American Journal of Applied Sciences 6 (12): 1988-1994, 2009

ISSN 1546-9239

(C) 2009 Science Publications

\title{
The Effect of Bone Properties due to Skeletal Diseases on Stability of Cementless Hip Stems
}

\author{
${ }^{1,2}$ Mohammed Rafiq Abdul Kadir and ${ }^{2}$ Nazri Kamsah \\ ${ }^{1}$ Medical Implant Technology Group, \\ Faculty of Biomedical Engineering and Health Sciences, \\ University Technology Malaysia, 81310 UTM Skudai, Johor, Malaysia \\ ${ }^{2}$ Faculty of Mechanical Engineering, \\ University Technology Malaysia, 81310 UTM Skudai, Johor, Malaysia
}

\begin{abstract}
Problem statement: There are two types of implant fixation in hip joint replacementcemented and cementless. The cemented types are, in general, more popular due to concerns of possible inability of cementless implants achieving maximum primary stability for bone integration. The concern is more significant in cases where there are major losses of cancellous bone stock and thinning of the trabeculae due to osteoporosis. Approach: Three Computed Tomography (CT) images of human hip joints were obtained from a hospital. The first patient showed osteoporotic condition based on DEXA scan of the bone. The second was registered for total hip replacement due to significant deterioration of the cartilage covering the bone ends. The third dataset was from a patient with no reported skeletal diseases and was used as control. Three dimensional models of the femora were reconstructed from the CT images and hip arthroplasty using cementless stem was simulated. Finite element method was used to analyze the stability of the implant through a specialized algorithm to measure micromotion at the bone-implant interface. Bone properties were assigned on an elementby element basis and loads simulating stair climbing were used. Results: Hip stems fixed in the control and osteoarthritic femoral model showed minimum interface micromotion. For the osteoporotic bone there is a progressive reduction in surface area feasible for bone in growth. Conclusion/Recommendations: Bone quality affects the stability of femoral components used in hip replacement and therefore the bone-implant integration potential. Cementless hip stem should not be used in patients with osteoporotic condition as the deterioration of bone tissues lead to an increase in interface micromotion.
\end{abstract}

Key words: Hip joint replacement, cementless stem, bone quality, osteoporosis, osteoarthritis, finite element analysis

\section{INTRODUCTION}

Total replacement of the hip joint is one of the most common procedures in orthopaedic surgery. Even though the procedure is regarded by many as one of the most successful, complications may still occur postsurgery ${ }^{[1-8]}$. Loosening of the femoral component is one of the failures associated with thigh pain with revision surgery the only option to relieve pain and regain mobility ${ }^{[9-11]}$. Micromotion at the interface between the implant and the bone is thought to be the reason behind this major complication. If the magnitude exceeded a certain threshold limit, fibrous tissue layer would form at the interface at the expense of bone formation ${ }^{[12-14]}$. Interface micromotion would increase as a result and the vicious cycle continues until the implant eventually loosened.

There are many factors that can cause excessive interface micromotion such as implant design, surgical technique, types of fixation, unphysiological loading condition and bone and joint diseases ${ }^{[15-18]}$. Skeletal diseases such as osteoporosis and osteoarthritis alter the bone's properties and its ability to provide adequate fixation. Osteoarthritic changes mainly concentrated at the articulating surface of the joint where the soft cartilage tissues deteriorates due to age or injury ${ }^{[19]}$. The properties of bone further away from the articulating joint may or may not change. Osteoporosis, on the other hand, is a skeletal disorder characterized by a significant loss of bone stock and structural

Corresponding Author: Mohammed Rafiq Abdul Kadir, Medical Implant Technology Group,

Faculty of Biomedical Engineering and Health Sciences, University Technology Malaysia, Malaysia 
deterioration of bone tissues ${ }^{[20-22]}$. The bone becomes fragile and more easily fractured under a sudden load due to the thinning of the trabeculae.

Osteoarthritis is the most common cause of hip disease leading to primary hip replacement because it causes pain and can severely reduced mobility. However, patients requiring hip arthroplasty also sometimes suffer from osteoporosis ${ }^{[19]}$. As osteoporotic bone is significantly weaker than healthy ones, it affects the decision in terms of selection of a suitable hip implant for replacement. It has been suggested that patients with osteoporosis would be better off having cemented hip stems to provide strong primary fixation ${ }^{[20,22]}$. However, it was also reported that cementless stems were also reliable for elderly patients with poor bone stock ${ }^{[23]}$.

The study on bone quality for replacement joint surgery is important as it has been found to influence the extent of stress-shielding-a situation where bone resorbs in areas where it is not adequately loaded. This is evident where severe bone loss was found in poorer quality bones than in healthy ones ${ }^{[24,25]}$. In terms of achieving primary stability, it is unknown if the weaker bone stock causes more micromotion and instability of the replaced hip. In general, stronger bone is preferred for stability with cemented stems regarded as the gold standard for fixation ${ }^{[25]}$. Cementless fixation is less popular as it has been assumed to provide less fixation strength. The use of cementless stems in osteoporotic bone for replacement surgery has therefore been avoided due to the weak condition of the bone.

In this study, finite element method was used to analyze the effect of bone quality on interface micromotion and therefore stability of a replaced hip joint. Two computed tomography (CT) image datasets of hip joints with different skeletal diseases were used in the analysis and compared with the ones reconstructed from a CT dataset of a normal healthy femoral bone. A specialized validated algorithm which calculates micromotion at the bone-implant interface was used to predict instability of the femoral component.

\section{MATERIALS AND METHODS}

CT datasets from two patients suffering from ostoearthritic of the hip joint were used in this study. The Young Adult T-score of one of the patients, which is the World Health Organization (WHO) criterion for osteoporosis, also showed marked osteoporosis in all regions of the femur. Another CT dataset of a normal healthy femur was taken as control. Three dimensional models of the three femoral bones were reconstructed and meshed with solid tetrahedrals.

After reconstruction of the bone models, the size of a suitable femoral stem was identified for each bone model using a fit-and-fill concept. The size of the canal was measured to determine the diameter of the stem to be used. The best size that fits the normal bone was $135 \mathrm{~mm}$, whilst the osteoporotic femoral model had a canal size of 150-15 mm larger than the normal bone due to the thinning of the cortical bone (Fig. 1). The osteoarthritic femoral model had a smaller canal of $120 \mathrm{~mm}$. Generic hip stem models with cylindrical features were developed with the diameter that matched the three femoral bones. The implants were then positioned inside their respective bone canals and the necks of the implants were angulated according to the anteversion angles of their original intact bones. The stems were assigned a linear isotropic material properties resembling titanium alloy (110 GPa), whilst the properties of the bone was assigned according to the grey level values of the CT dataset. An in-house algorithm was used to correlate the grey-level of the CT images using the apparent density through cubic correlation proposed by Carter and Hayes ${ }^{[26]}$ :

$\mathrm{E}=\mathrm{c} \rho^{3}$

where, $\mathrm{c}=3790 \mathrm{MPa} \cdot \mathrm{g}^{-3} \mathrm{~cm}^{9}$. This relationship was based on the assumption that cancellous and cortical bones are simply at different ends of a continuous spectrum.

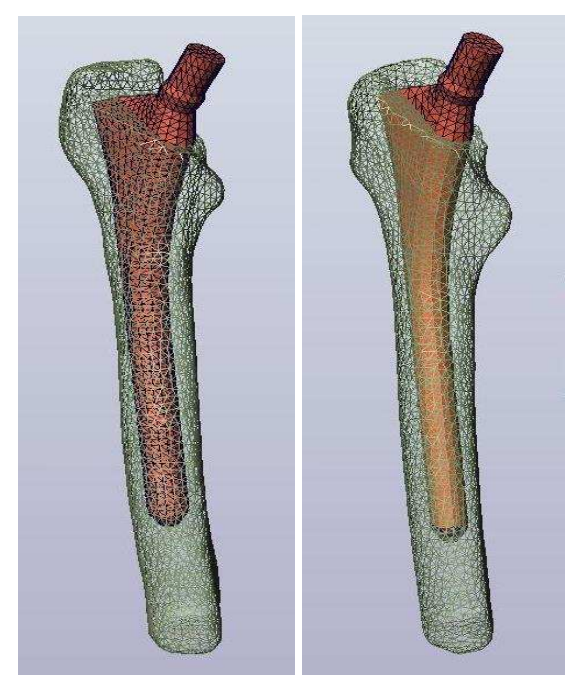

Fig. 1: Outlined view of the osteoporotic femur with a $150 \mathrm{~mm}$ diameter straight cylindrical stem inside (left) and a normal femoral bone with $135 \mathrm{~mm}$ stem (right) 
The three simulated arthroplasty models were loaded using a published dataset which includes muscle forces (Fig. 2). The dataset was obtained from the work of Duda $^{[27]}$ in which muscle and joint forces for two physiological activities, walking and stair climbing, were measured using telemetry (Table 1).

An in-house experimentally validated computer algorithm ${ }^{[28]}$ was used to measure micromotion at the interface and predict instability of the stem. This algorithm calculates the displacement of the stem relative to the endosteal surface of the bone by subtracting displacement values between corresponding nodes at the interface. Non-linear contact analysis was used by assigning target and contact surfaces between the individual parts of the model. The constraint associated with no penetration is implemented by transforming the degrees of freedom of the contact node and applying a boundary condition to the normal displacement.

In order to check for instability, interfacial bone loss was simulated in areas where micromotion exceeded the threshold micromotion limit of 100 microns. The new models with simulated interfacial bone loss were then loaded in the same stair climbing mode. The iterations were continued until either a stable state micromotion was achieved or loosening was predicted.

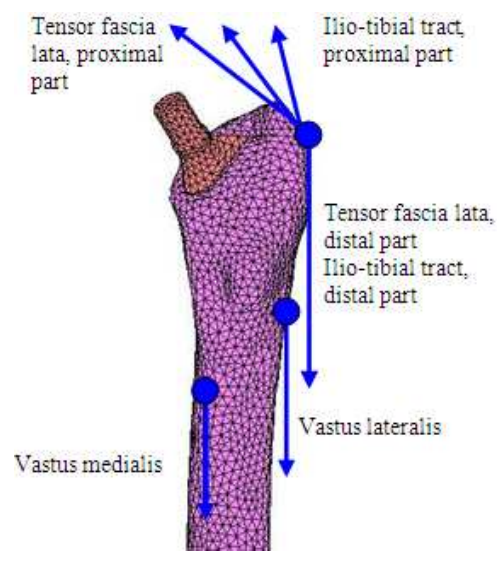

Fig. 2: Location of the muscles attachment used by Duda ${ }^{[28,29]}$

Table 1: Maximum loading configurations in stair climbing activity used by Duda ${ }^{[27]}$

\begin{tabular}{lrrr}
\hline Force $(\mathrm{N})$ & \multicolumn{1}{c}{$\mathrm{X}$} & \multicolumn{1}{c}{$\mathrm{Y}$} & \multicolumn{1}{c}{$\mathrm{Z}$} \\
\hline Joint contact force & -476.4 & -486.8 & 1898.3 \\
Abductor & 563.1 & 231.4 & -682.1 \\
Ilio-tibial tract, proximal part & 84.4 & -24.1 & -102.8 \\
Ilio-tibial tract, distal part & -4.0 & -6.4 & 135.0 \\
Tensor fascia lata, proximal part & 24.9 & 39.4 & -23.3 \\
Tensor fascia lata, distal part & -1.6 & -2.4 & 52.2 \\
Vastus Lateralis & -17.7 & 180.0 & 1085.3 \\
Vastus Medialis & -70.7 & 318.1 & 2145.8
\end{tabular}

The Canal Flare Index (CFI) was calculated for the three bone models using the technique proposed by previous researchers ${ }^{[29]}$. The ratio of the canal diameter at two locations $-20 \mathrm{~mm}$ above the lesser trochanter and the isthmus-was calculated from the anteroposterior view.

\section{RESULTS}

Figure 3 shows slice-through models of the three bones, together with their corresponding stiffness distribution. There was a large decrease in stiffness in the osteoporotic bone as well as significant reduction in thickness of the cortex compared to the healthy bone. The osteoarthritic femur showed a high stiffness value in the medial and lateral cortex.

The values of the Canal Flare Index (CFI) are shown in Table 2. The VHP bone had CFI of 3.6, the osteoporotic bone had CFI of 2.3 and the osteoarthritic had CFI of 6.2.

Figure 4 shows contour plots of micromotion under simulated physiological stair climbing using the validated algorithm. Figure 4 shows larger micromotion at the bone-implant interface for the osteoporotic model compared to the control VHP bone model.

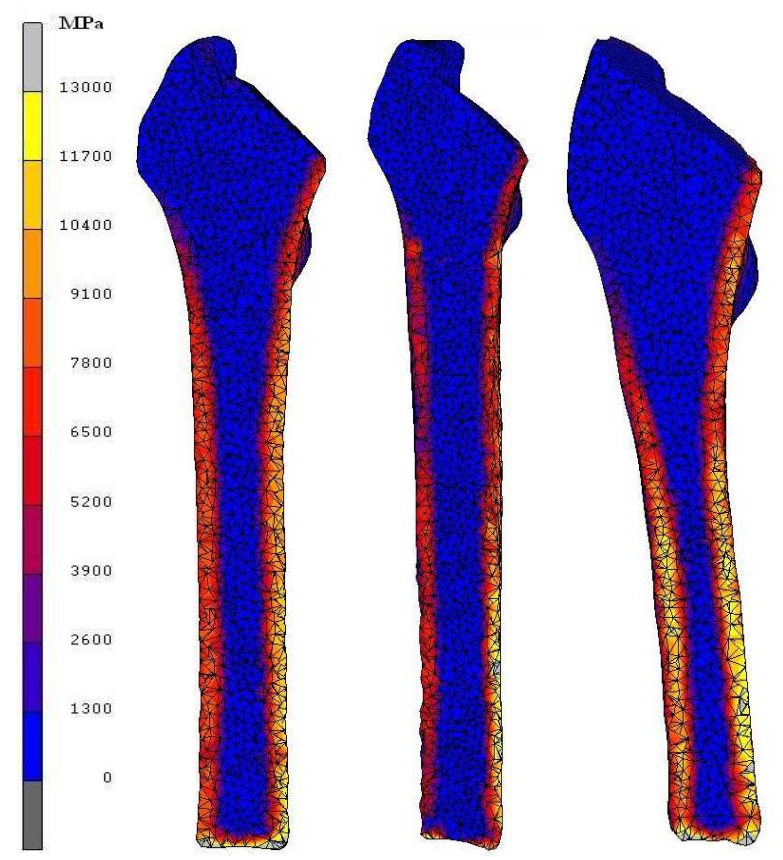

Fig. 3:Young's Modulus of the three bones-normal (left), osteoporosis (middle) and osteoarthritic (right) 
Table 2: The canal flare index of the three bones

\begin{tabular}{ll} 
Normal & 3.6 \\
Oateoporotic & 2.3 \\
Osteoarthritic & 6.2 \\
\hline
\end{tabular}

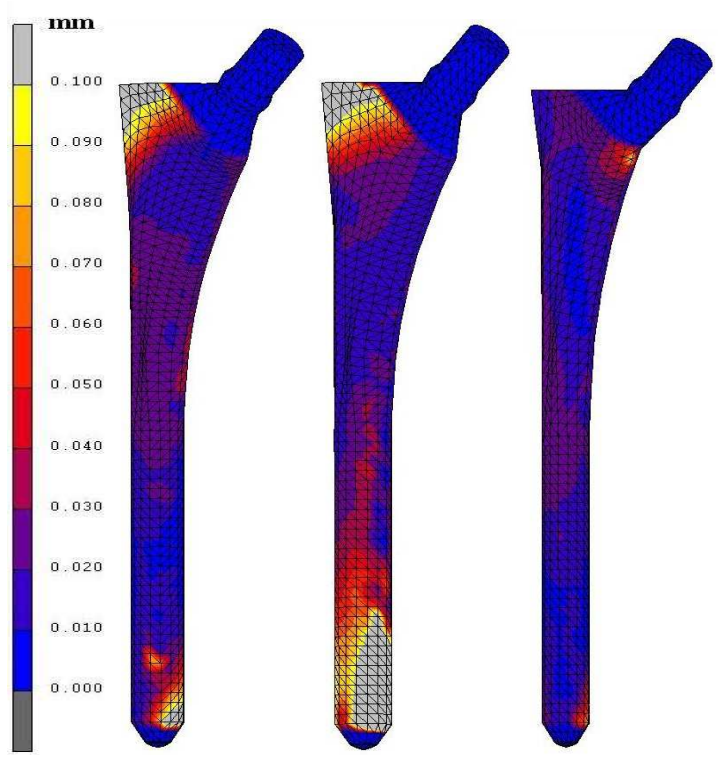

Fig. 4: Micromotion results for the hip stem implanted in the normal bone (left), osteoporotic (middle) and osteoarthritic (right)

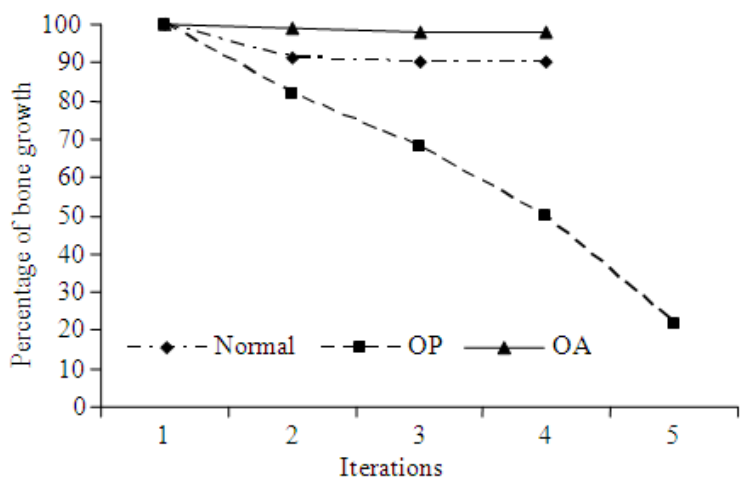

Fig. 5: The reduction of surface area feasible for bone growth

The distribution of micromotion was, however, similar between the two models, with larger micromotion observed at the proximal and distal part of the stem. The difference in magnitude of micromotion between osteoporotic and normal bones ranged from a factor of 3 to a maximum of about 4 . The maximum micromotion found for the osteoporotic model also reached 250 microns.

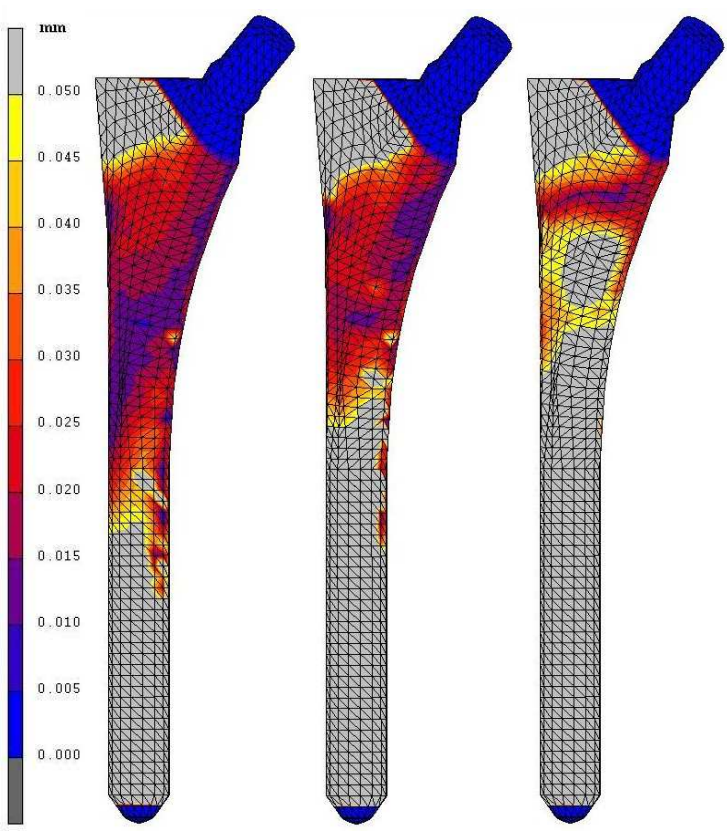

Fig. 6: Micromotion for the hip stem relative to the osteoporotic bone for the 2nd, 3rd and 4th iteration showing an increase insurface area unfeasible for bone growth

The results of the surface area unfeasible for bone ingrowth are shown in Fig. 5. The results showed that the cementless straight cylindrical stem was unstable in the osteoporotic bone; the surface area unfeasible for bone ingrowth increased from $18 \%$ in the first iteration to $78 \%$ in the fourth iteration. Figure 6 shows the contour plots of micromotion for the osteoporotic model showing the predicted instability of the stem.

\section{DISCUSSION}

Osteoporosis is a major skeletal disorder characterized by the structural deterioration of bone tissues. This includes significant loss of cancellous bone stock, thinning of the trabeculae and thinning of the outer cortex. This is in stark contrast to osteoarthritis where the apparent density seemed to be increasing in the trabecular region resulting in greater stiffness, yield strength and energy absorption ${ }^{[19]}$. Special attention is therefore required in cases involving hip replacement of the bone with osteoporotic condition. Due to the significant alteration in the material and mechanical properties of the cortical and cancellous bone, primary fixation may not be as strong as in those without osteoporosis. Cementless approach to hip arthroplasty may not be appropriate and has been very much avoided in this situation. 
To the author's knowledge, there are no published reports comparing the primary stability of prostheses inside normal bone and bone with osteoporotic condition using finite element method. However, there are several reports showing preference of good bone quality to poor bone stock. In a follow-up study by Kim and $\mathrm{Kim}^{[30]}$, the authors reported that the AML stems used in younger patients had much lower frequency of loosening and revision rates compared to older patients. They have attributed the success to good bone quality, among other things. Other researchers ${ }^{[31]}$ reported that femoral prosthesis loosening after seven years could be predicted by bone quality at the time of implantationwith loosening more likely if the bone quality was poor. A retrieval study ${ }^{[32]}$ on various types of cemented and cementless stems showed that the extent of bone loss depended on the density of the bone; the less dense the bone was pre-operatively, the greater the extent of bone loss. This trend was also found by Kerner et al. ${ }^{[24]}$ in his study on bone remodeling using a straight cylindrical AML cementless prosthesis.

In this study, three CT datasets of the hip joint were obtained from a local hospital. Two of them were registered for arthroplasty due to osteoarthritis of the hip joint. One of them also suffered from osteoporosis as proven by the DEXA scan. Our calculated CFI showed that the osteoporotic bone was of type $\mathrm{C}$ with stovepipe characteristics, whilst the osteoarthritic model had a champagne-fluted appearance of type A bone. The normal bone was categorized as type B bone with CFI between 3.0-4.7 $7^{[23]}$.

For the osteoporotic bone, surgeons may have less of a choice in terms of a suitable hip stem to register compared to the one without osteoporosis. This is because primary stability has to be achieved for proper bone healing process. Unstable femoral components will result in the formation of fibrous tissue layer at the bone-implant interface ${ }^{[12,13]}$. The fibrous layer will increase micromotion at the interface with eventual loosening of the component.

In order to achieve primary stability, cemented stems are normally used. It has been regarded as the gold standard for implant fixation. However, cementless stems are gaining popularity due to the conservation of bone stock, with reported long-term success rate ${ }^{[10,11]}$. Rigid fixation is achieved through micro and macrofeatures in the proximal or distal end, or both.

Although there are many reported success on the use of cementless stems in hip arthroplasty, many are still skeptical on its use especially in patients with poor bone stock $^{[17-19]}$. Our finite element analysis showed that the osteoporotic bone, which had reduced bone stiffness and thinning of the cortex on the anterior and posterior sides showed significantly larger micromotion particularly in the distal area. Minimum contact with endosteal cortices and reduced cortical stiffness could be the reason for this. The FE study also showed that patients who have had cementless hip replacement due to osteoarthritis with stronger bone stock should have fewer problems in terms of stability compared to those with poorer bone stock.

\section{CONCLUSION}

The stability of a cementless straight cylindrical femoral stem in osteoporotic femur was compared with two other femoral models with stronger bone stock. An in-house experimentally validated micromotion algorithm was used to predict instability of the femoral stems under simulated stair-climbing activity. The results showed that straight cylindrical stem using a cementless approach was not suitable for replacing a hip joint in an osteoporotic femur.

\section{ACKNOWLEDGEMENT}

This project has been supported by the Government of Malaysia.

\section{REFERENCES}

1. Bauer, T.W. and J. Schils, 1999. The pathology of total joint arthroplasty, II. Mechanisms of implant failure. Skeletal Radiol., 28: 483-497. DOI: 10.1007/s002560050552

2. Hanssen, A.D. and J.A. Rand, 1998. Instructional course lectures. American Academy of Orthopaedic Surgeons. Evaluation and treatment of infection at the site of a total hip or knee arthroplasty. J. Bone Joint Surg. Am., 80: 910-922. DOI: 10.1007/978-1-59259-975-2_52

3. Gristina, A.G. and J.W. Costerton, 1985. Bacterial adherence to biomaterials and tissue: The significance of its role in clinical sepsis. J. Bone Joint Surg. Am., 67: 264-273. DOI: 10.1016/02782391(85)90028-X

4. Garvin, K.L. and A.D. Hanssen, 1995. Current concepts review. Infection after total hip arthroplasty: Past, present and future. J. Bone Joint Surg. Am., 77: 1576-1588. http://www.ejbjs.org/cgi/reprint/77/10/1576.pdf

5. Barrack, R.L. and W.H. Harris, 1993. The value of aspiration of the hip joint before revision total hip arthroplasty. J. Bone Joint Surg. Am., 75: 66-76. http://www.ejbjs.org/cgi/content/abstract/75/1/66 
6. Wright, T.M. and S.B. Goodman, 1996. Implant Wear: The Future of Total Joint Replacement. III. 1st Edn., American Academy of Orthopaedic Surgeons Publications, ISBN: 0892031484, pp: 116.

7. Sutula, L.C., J.P. Collier, K.A. Saum, B.H. Currier and J.H. Currier et al., 1995. Impact of gamma sterilization on clinical performance of polyethylene in the hip. Clin Orthop., 319: 28-40. http://journals.lww.com/corr/Abstract/1995/10000/ The_Otto_Aufranc_Award_Impact_of_Gamma.4. aspx

8. McKellop, H.A., 1995. Wear Modes, Mechanisms, Damage and Debris: Separating Cause from Effect in the Wear of Total Hip Replacements. In: Total Hip Revision Surgery. Galante J.O., A.G. Rosenberg and J.J. Callaghan (Eds.). Raven Press, New York, pp: 21-39.

9. Brodner, W., P. Bitzan, F. Lomoschitz, P. Krepler, R. Jankovsky et al., 2004. Changes in bone mineral density in the proximal femur after cementless total hip arthroplasty a five-year longitudinal study. J. Bone Joint Surg. Br., 86-B: 20-26. DOI: 10.1302/0301-620X.86B1.14637

10. Chen, C.H., C.H. Shih and C.C. LinCheng, 1998. Cementless Roy-Camille femoral component. Arch. Orthop. Trauma Surg., 118: 85-88. DOI: 10.1007/s004020050318

11. Chiu, K.Y., W.M. Tang, T.P. Ng, K.C. Poon, W.Y. Ho and K.M. Lee, 2001. Cementless total hip arthroplasty in young Chinese patients a comparison of 2 different prostheses. J. Arthroplasty, $\quad 16$ : $863-870 . \quad$ DOI: 10.1054/arth.2001.25505

12. Pilliar, R.M., J.M. Lee and C. Maniatopoulos, 1986. Observations on the effect of movement on bone ingrowth into porous-surfaced implants. Clin. Orthop. Related Res., 208: 108-113. DOI: 10.1054/arth.2001.25505

13. Pilliar, R.M., 1991. Quantitative Evaluation of the Effect of Movement at a Porous Coated ImplantBone Interface. The Bone-Biomaterial Interface, Davies, J.E. (Ed.), 1st Edn., University of Toronto Press, pp: 380-387.

14. Simmons, C.A., N. Valiquette and R.M. Pilliar, 1999. Osseointegration of sintered porous-surfaced and plasma spray-coated implants: An animal model study of early post implantation healing response and mechanical stability. J. Biomed. Mater. Res., 47: 127-138.

http://www.mie.utoronto.ca/labs/mechanobio/conte nt/publications/Simmons_1999_JBMR_Implant_su rface_design.pdf
15. Adam, F., D.S. Hammer, S. Pfautsch and K. Westermann, 2002. Early failure of a press-fit carbon fiber hip prosthesis with a smooth surface. J. Arthroplasty, 17: 217-224. DOI: 10.1054/arth.2002.30285

16. Karrholm, J., C. Anderberg, F. Snorrason, J. Thanner, N. Langeland, H. Malchau and P. Herberts, 2002. Evaluation of a femoral stem with reduced stiffness a randomized study with use of radiostereometry and bone densitometry. J. Bone Joint Surg., 84: 1651-1658. http://www.ejbjs.org/cgi/content/abstract/84/9/1651

17. Haddad, R.J., S.D. Cook and M.R. Brinker, 1990. A comparison of 3 varieties of noncemented porous coated hip replacement. J. Bone Joint Surg. Br., 72: 2-8. http://www.jbjs.org.uk/cgi/reprint/72B/1/2.pdf

18. Jacobsson, S.A., K. Djerf, J. Gillquist, S. Hammerby and I. Ivarsson, 1993. A prospective comparison of butel and PCA hip arthroplasty. J. Bone Joint Surg. Br., 75: 624-629.

http://www.jbjs.org.uk/cgi/content/abstract/75$\mathrm{B} / 4 / 624$

19. Li, B. and R.M. Aspden, 1997. Material properties of bone from the femoral neck and calcar femorale of patients with osteoporosis or osteoarthritis. Osteoporos Int., 7: 450-456. DOI: 10.1007/s001980050032

20. Dorr, L.D., Z. Wan and T. Gruen, 1997. Functional results in total hip replacement in patients 65 years and older. Clin. Orthop. Relat. Res., 1: 143-151. DOI: 10.1097/00003086-199703000-00020

21. Harris, W.H., 1997. Options for primary femoral fixation in total hip arthroplasty: Cemented stems for all. Clin. Orthop. Relat. Res., 1: 118-123. DOI: 10.1097/00003086-199711000-00013

22. Haber, D. And S.B. Goodman, 1998. Total hip arthroplasty in juvenile chronic arthritis a consecutive series. J. Arthroplasty., 13: 259-265. DOI: 10.1016/S0883-5403(98)90170-X

23. Healy, W.L., 2002. Hip Implant selection for Total Hip Arthroplasty in elderly patients. Clin. Orthop. Relat. $\quad$ Res., 405: 54-64. http://journals.lww.com/corr/Abstract/2002/12000/Hi p_Implant_Selection_for_Total_Hip_Arthroplasty.7.a $\operatorname{spx}$

24. Kerner, J., R. Huiskes, G.H. van Lenthe, H. Weinans, B. van Rietbergen, C.A. Engh and A.A. Amis, 1999. Correlation between pre-operative periprosthetic bone density and post operative bone loss in THA can be explained by strain-adaptive remodeling. J. Biomech., 32: 695-703. http://yp.bmt.tue.nl/pdfs/635.pdf 
25. Ohsawa, S., K. Fukuda, S. Matsushita, S. Mori, H. Norimatsu and R. Ueno, 1998. Middle-term results of anatomic medullary locking total hip arthroplasty. Arch. Orthop. Trauma Surg., 118: 14-20. DOI: $10.1007 / \mathrm{s} 004020050302$

26. Carter, D. and W. Hayes, 1978. The compressive behavior of trabecular bone. J. Biomech., 11: 209. DOI: 10.1016/0021-9290(78)90016-7

27. Duda, G.N., M. Heller, J. Albinger, O. Schulz, E. Schneider and Claes, 1998. Influence of muscle forces on femoral strain distribution. J. Biomech., 31: 841-846. DOI: 10.1016/S0021-9290(98)00080-3

28. Abdul-Kadir, M.R., U. Hansen, R. Klabunde, D. Lucas and A. Amis, 2008. Finite element modeling of primary hip stem stability: The effect of interference fit. J. Biomech., 41: 587-594.

29. Noble, P.C., J.W. Alexander, L.J. Lindahl, D.T. Yew, W.M. Granberry and H.S. Tullos, 1998. The anatomic basis of femoral component design. Clin. Orthop. Relat. Res., 235: 148-165. DOI: 10.1097/00003086-198810000-00015
30. Kim, Y.H. and V.E.M. Kim, 1994. Cementless porous-coated anatomic medullary locking total hip prostheses. J. Arthroplasty, 9: 243-252. DOI: 10.1016/0883-5403(94)90078-7

31. Krischak, G.D., N.J. Wachter, T. Zabel, G. Suger and A. Beck et al., 2003. Influence of preoperative mechanical bone quality and bone mineral density on aseptic loosening of total hip arthroplasty after seven years. Clin. Biomech., 18: 916-923. DOI: 10.1016/S0268-0033(03)00175-X

32. Maloney, W.J., C. Sychterz, C. Bragdon, T. McGovern and M. Jasty et al., 1996. Skeletal response to well fixed femoral components inserted with and without cement. Clin. Orthop. Relat. Res., 333: 15-26. DOI: 10.1097/00003086-199612000-00003 